\title{
Efeito larvicida de óleos essenciais de plantas medicinais sobre larvas de Aedes aegypti L. (Diptera:Culicidae)
}

\section{Larvicidal effect of essential oils of medicinal plants against larvae of Aedes aegypti $\mathbf{L}$. (Diptera: Culicidae)}

\author{
Toshik Iarley da Silva ${ }^{l *}$, Antônio Carlos Leite Alves ${ }^{2}$, Francisco Roberto de Azevedo ${ }^{3}$, Cláudia Araújo Marco ${ }^{4}$, Hernandes \\ Rufino dos Santos ${ }^{5}$, William Santana Alves ${ }^{6}$
}

\begin{abstract}
Resumo: O Aedes aegypti L. é o mosquito mais relevante em termos de inseto de importância para a saúde pública no mundo, pois é transmissor de vírus que causam várias doenças, dentre elas a dengue. Na procura de larvicidas alternativos, os óleos essenciais extraídos de plantas medicinais têm demonstrado alta eficiência. Com isso, objetivou-se com avaliar o potencial larvicida de óleos essenciais, extraídos de espécies medicinais, sobre larvas de A. aegypti. O experimento foi realizado nos Laboratórios de Tecnologia de Produtos e de Entomologia Agrícola da Universidade Federal do Cariri (UFCA), na cidade de Crato, Ceará. Os óleos essenciais de alfazema (Hyptis suaveolens); gonçalo-alves (Astronium fraxinifolium0; alecrim de tabuleiro (Lippia Microphylla); mussambê (Cleome spinosa); marmeleiro (Croton sonderianus); aroeira (Myracrodruon urundeuva); velame (Croton heliotropiifolius) e candeeiro (Vanillosmopsis arborea) foram extraídos pelo método de hidrodestilação em aparelho tipo Clevenger. Os óleos foram emulsionados com Dimetilsulfóxido 2\% (DMSO) e diluídos para a concentração de 100 ppm (partes por milhão), utilizando-se o delineamento inteiramente casualisado com nove tratamentos (consistindo nas soluções de 100 ppm de cada óleo mais a solução controle com água e DMSO) e quatro repetições, utilizandose dez larvas para cada tratamento, sendo avaliado o número de larvas mortas. Observou-se que todos os óleos essenciais apresentaram efeito larvicida, porém os de candeeiro e de alfazema foram os que se destacaram.
\end{abstract}

Palavras-chave: Biolarvicida; Dengue; Hyptis suaveolens; Vanillosmopsis arborea

\begin{abstract}
Aedes aegypti L. is the most relevant mosquito in terms of public health importance of the world, because it's the virus transmitter that causes many diseases, among this dengue. Looking for alternatives larvicides, the essential oils extracted from medicinal plants have demonstrated high efficiency. Thereby, it was objectified in this work to evaluate the larvicide potential of some essential oils, extracted from medicinal species, against A. aegypti larvae. The experiment was realized at the Laboratory of Products Technology and Agricultural Entomology of the Federal University of Cariri (UFCA), in city of Crato, Ceará state. The essential oils alfazema (Hyptis suaveolens); gonçalo-alves (Astronium fraxinifolium); alecrim de tabuleiro (Lippia Microphylla); mussambê (Cleome spinosa); marmeleiro (Croton sonderianus); aroeira (Myracrodruon urundeuva); velame (Croton heliotropiifolius) e candeeiro (Vanillosmopsis arborea) was extracted by hydrodistillation method in apparatus like Clevenger type. The oils were emulsified with dimethyl sulfoxide 2\% (DMSO) and diluted to a concentration of $100 \mathrm{ppm}$ (parts per million), utilizing design completely randomized with nine treatments (that consisted in the solutions of $100 \mathrm{ppm}$ of each oil plus control solution with water and DMSO) and four repetitions, utilizing ten larvae for each treatment, being evaluated the number of dead larvae. Was calculated the mortality average by Tukey test with $5 \%$ of probability and the efficiency. It was observed that all the essential oils presented larvicide effect, but $V$. arborea and $H$. suaveolens was the ones which was highlighted.
\end{abstract}

Key words: Biolarvicide; Dengue fever; Hyptis suaveolens; Vanillosmopsis arborea

\footnotetext{
*Autor para correspondência

Recebido para publicação em 06/01/2017; aprovado em 08/06/2017

${ }^{1}$ Engenheiro agrônomo, mestrando em Agronomia (UFPB), Universidade Federal da Paraíba, Areia-PB, iarley.toshik@gmail.com.

${ }^{2}$ Engenheiro agrônomo, mestrando em Agronomia (UFPB), Universidade Federal da Paraíba, Areia-PB, carlos.ufca@ yahoo.com.br

${ }^{3}$ Engenheiro agrônomo, Doutor em Fitotecnia (UFC), Professor Adjunto da Universidade Federal do Cariri, Crato-CE, roberto.azevedo@ufca.edu.br

${ }^{4}$ Engenheira agrônoma, Doutora em Fitotecnia (UFC), Professora Associada da Universidade Federal do Cariri, Crato-CE, claudia.marco@ufca.edu.br

${ }^{5}$ Engenheiro agrônomo, Doutor em Fitotecnia (UFC), Crato-CE, hernandes.rufino@ufca.edu.br

${ }^{6}$ Biólogo, Especialista em Educação Ambiental (URCA), Crato-CE, williamsantana_@hotmail.com
} 


\section{INTRODUÇÃO}

Os mosquitos são os mais relevantes em termos de insetos de importância para a saúde pública que transmitem um grande número de doenças como a dengue, a chikungunya, encefalite japonesa, filariose e a malária, causando milhões de mortes todos os anos (SRITABUTRA; SOONWERA, 2013).

Dentre os vários mosquitos vetores de doenças, destacase o Aedes aegypti L., mosquito originário da África, onde existem populações selvagens e domésticas. Originalmente descrito no Egito, o que lhe conferiu seu nome específico. $\mathrm{Na}$ atualidade, a espécie tem distribuição mundial. É um mosquito adaptado ao ambiente urbano e utiliza os recipientes mais frequentes no domicílio ou peridomicílio (tanques de armazenamento de água e vasilhames temporários, dentro e fora das casas, como potes, barris, pneumáticos usados, latas, garrafas e vasos de plantas) para o desenvolvimento de sua fase larvária (BRAGA; VALLE, 2007).

Dentre os vários tipos de mosquitos que vivem em regiões tropicais e subtropicais, o A. aegypti é o vetor conhecido por ser transmissor de vírus causadores de doenças como febre amarela urbana e dengue, sendo responsáveis por um número de morbidade e mortalidade em todo o mundo, devido aos seus graves sintomas dessas doenças (SILVA et al., 2008).

O controle do mosquito utilizando inseticidas, temefós, malathion e fenitrothion, constitui a principal medida adotada pelos Programas de Saúde Pública. Entretanto, em diferentes partes do mundo e no Brasil, tem sido registrada resistência desse díptero aos inseticidas convencionais (FURTADO et al., 2005; PROPHIRO et al., 2008; PORTO et al., 2008; PROPHIRO et al., 2011).

A primeira informação sobre o desenvolvimento de resistência do A. aegypti aos inseticidas data de 1950 e referese a uma população do mosquito originária do Caribe, em relação aos organofosforados. Posteriormente, foram surgindo outros trabalhos mostrando a ocorrência de resistência a organofosforados e piretróides, em regiões tropicais e subtropicais (GUIRADO; BICUDO, 2009).

Lima et al. (2006), em estudos sobre a resistência ao temefós no estado do Ceará destacam que as amostras de $A$. aegypti provenientes dos bairros de Fortaleza apresentaram um baixo porcentual de mortalidade quando expostas a este produto. Resultados semelhantes foram também para amostras de $A$. aegypti procedentes dos municípios de Juazeiro do Norte, Crato e Barbalha, evidenciando que a resistência mostra-se também difundida no interior do Estado.

Para contornar estes problemas, é necessário identificar novas alternativas de controle com diferentes modos de ação a fim de aumentar as opções disponíveis de inseticidas de uso na saúde pública. $\mathrm{O}$ inseticida ideal deve ser eficaz, ecologicamente correto, sustentável e rentável e apresentar baixa toxicidade aos mamíferos. Além disso, não deverão alterar significativamente as características da água (DIAS; MORAES, 2014).

Algumas substâncias botânicas têm atividade inseticida conhecida, tais como, piretrinas, rotenona, nicotina, cevadina, veratridina, rianodina quassinóides, azadiractina e bioinseticidas voláteis. Estes últimos são, normalmente, óleos essenciais presentes nas plantas aromáticas (CORRÊA; SALGADO, 2011).
Os constituintes dos óleos essenciais são principalmente compostos lipofílicos que atuam como toxinas, impedimentos de alimentação e oviposição para uma ampla variedade de insetos pragas (KOUL et al., 2008), sendo reconhecidos como importantes recursos naturais de inseticidas porque alguns são seletivos, biodegradáveis, não-tóxicos e têm alguns efeitos sobre organismos alvo (SRITABUTRA; SOONWERA, 2013).

Inseticidas baseados em plantas parecem não ter nenhum efeito nocivo sobre as populações não-alvo, além de ser disponível em muitas partes do mundo mais afetada pelas doenças transmitidas por mosquitos. Eles agem interferindo com o crescimento e a reprodução da praga e são eficazes contra diferentes fases do seu crescimento (SILVA et al., 2008).

Muitas plantas podem ser utilizadas com efeito larvicida sobre o A. aegypti. Dentre elas, destacam-se Piper marginatum (AUTRAN et al., 2009), Sapindus saponaria (BARRETO et al., 2006), Dendropanax morbifera (CHUNG et al., 2009), Citrus sinensis (EL-AKHAL et al., 2015), Copaifera reticulata (GERIS et al., 2008), Mentha piperita (KUMAR et al., 2011), Lippia sidoides (LIMA et al., 2013), Piper aduncum (OLIVEIRA et al., 2013), Anacardium humile (PORTO et al., 2008), dentre outras.

Com vista nisso, objetivou-se avaliar o efeito larvicida de óleos essenciais de plantas medicinais no controle de larvas de A. aegypti em condições de laboratório.

\section{MATERIAL E MÉTODOS}

Os óleos essenciais foram extraídos de folhas de alfazema-brava [Hyptis suaveolens (L.) Poit.]; gonçalo-alves [Astronium fraxinifolium Schott]; alecrim de tabuleiro [Lippia Microphylla Cham.]; mussambê [Cleome spinosa Jacq.]; marmeleiro [Croton sonderianus Muell. Arg.]; aroeira [Myracrodruon urundeuva (Allemão) Engl.]; e velame [Croton heliotropiifolius Kunth], que foram colhidas na cidade de Lavras da Mangabeira, Ceará. Caules de candeeiro [Vanillosmopsis arborea Baker] foram colhidos no município de Crato, Ceará.

Todos os materiais vegetais foram armazenados em sacos de cor escura e levados para o Laboratório de Tecnologia de Produtos (LTP) do Centro de Ciências Agrárias e da Biodiversidade da Universidade Federal do Cariri (CCAB/UFCA). Para obter melhor rendimento de óleo, o material foi triturado em pequenos pedaços com o auxílio de facões e tesouras de poda. Os óleos essenciais foram extraídos pelo método de hidrodestilação em aparelho tipo Clevenger conforme metodologia descrita por Alencar et al. (1984). Foram pesados $300 \mathrm{~g}$ de cada material e colocados submergidos em $2.000 \mathrm{ml}$ de água destilada em um balão de fundo chato com capacidade para $5.000 \mathrm{ml}$, estabelecendo um período de extração de 120 minutos.

Após o período de extração, o óleo essencial foi retirado do aparelho com o auxílio de uma pipeta de Pasteur e armazenado em eppendorf cobertos com papel alumínio em refrigerador doméstico.

Para a obtenção de ovos do inseto vetor foram utilizadas armadilhas do tipo ovitrampas, instaladas nos bairros: Lameiro, Seminário, Vila Alta e Centro da cidade de Crato, Ceará. Essas armadilhas foram constituídas de um vaso de planta de polipropileno preto, com capacidade para $400 \mathrm{ml}$, contendo no seu interior água e uma palheta de madeira 
prensada (tipo Eucatex), com dimensões de $3 \times 11 \mathrm{~cm}$, inserida na posição vertical da parede do vaso.

Após cinco dias de instalação das ovitrampas, as palhetas foram recolhidas e levadas para o Laboratório de Entomologia Agrícola do CCAB da Universidade Federal do Cariri (UFCA), onde, com o auxílio de lupa estereoscópica, foi realizada a contagem dos ovos do inseto.

Para a obtenção das larvas, as palhetas contendo os ovos coletados foram colocados em béqueres com capacidade de $2.000 \mathrm{ml} \mathrm{Em} \mathrm{seguida,} \mathrm{foi} \mathrm{adicionado} \mathrm{água} \mathrm{e} \mathrm{os} \mathrm{béqueres}$ levados para uma câmara climatizada do tipo B.O.D. (Biochemical Oxygen Demand) em condições controladas de temperatura de $25 \pm 1^{\circ} \mathrm{C}$, umidade relativa do ar de $70 \pm 10 \%$ e fotofase de 12 horas. Após a eclosão das larvas, as palhetas foram retiradas e as mesmas mantidas nessas condições e alimentadas com matéria orgânica até atingirem o terceiro instar.

O bioteste foi realizado no Laboratório de Entomologia Agrícola da UFCA. Para isso, cada óleo essencial foi diluído para uma concentração de $100 \mathrm{ppm}$. Foi pesado $10 \mathrm{mg}$ de cada óleo essencial em balança analítica (0,0001), adicionando-se $98 \mathrm{~mL}$ de água destilada e $2 \mathrm{ml}$ de Dimetilsufóxido (DMSO). A solução foi colocada em um balão volumétrico $(200 \mathrm{ml})$ e agitada manualmente até obterse uma solução homogênea.

Para cada tratamento foram utilizadas dez larvas entre o terceiro e quarto instar. As larvas foram retiradas do béquer onde estavam acondicionadas com o auxílio de uma pipeta de Pasteur, retirado o excesso de água e colocadas em copos de polietileno com capacidade de $50 \mathrm{ml}$. Logo em seguida, com o auxílio de uma pipeta volumétrica $(25 \mathrm{ml})$ foi colocado 25 $\mathrm{mL}$ da solução em cada copo que continha as larvas. Após 24, 48 e $72 \mathrm{~h}$ de exposição das larvas aos tratamentos, o número de larvas mortas foi registrado sendo consideradas mortas aquelas que não apresentavam movimento ou não respondiam aos estímulos com a pipeta de Pasteur.

A eficiência de mortalidade das larvas foi determinada em porcentagem por meio da equação 1 (ABBOTT, 1925)

$$
\mathrm{E}(\%)=\frac{\mathrm{Nc}-\mathrm{Nt}}{\mathrm{Nc}} \times 100
$$

Em que: $=$ Eficiência $; \mathrm{Nc}=$ Número de indivíduos vivos no tratamento controle; $\mathrm{Nt}=$ Número de indivíduos vivos tratados

O delineamento estatístico adotado foi o inteiramente casualizados (DIC) com nove tratamentos correspondendo aos óleos essenciais das espécies utilizadas (H. suaveolens, $A$. fraxinifolium, L. microphylla, $C$. spinosa, C. sonderianus, $M$. urundeuva, $C$. heliotropiifolius e de $V$. arborea) e água destilada mais DMSO (testemunha) e quatro repetições, com 10 larvas cada, totalizando 36 unidades experimentais. Os dados obtidos foram submetidos à análise de variância (ANOVA) e as suas médias comparadas pelo teste de Tukey a 5\% de probabilidade utilizando o Programa Operacional SISVAR-UFLA (FERREIRA, 2011).

\section{RESULTADOS E DISCUSSÃO}

As mais recentes investigações em vários países confirmam que alguns óleos essenciais de plantas não têm apenas a capacidade de repelir insetos, mas apresentam também ação inseticida através do contato direto ou pelas vias respiratórias dos insetos (CORRÊA; SALGADO, 2011).
Quando analisado o período de exposição de 24 horas das larvas aos óleos essenciais (Tabela 1), verificou-se que o óleo de $V$. arborea foi o que causou maior mortalidade às larvas, diferindo estatisticamente dos demais tratamentos, exceto de $H$. suaveolens. Todos os demais tratamentos, não diferiram estatisticamente da testemunha, no entanto, demostram atividade larvicida, exceto L. microphylla e $C$. spinosa. Com isso, percebe-se que os óleos essenciais de $V$. Arborea e $H$. suaveolens, são eficientes no controle das larvas do A. aegypti, neste primeiro período analisado.

Tabela 1. Mortalidade de larvas de A. aegypti e eficiência de óleos essenciais após períodos de exposição.

\begin{tabular}{lcccc}
\hline \multirow{2}{*}{ Espécies } & \multicolumn{4}{c}{ Mortalidade / Eficiência [E\%] } \\
\cline { 2 - 5 } V. arborea & $24 \mathrm{~h}$ & $48 \mathrm{~h}$ & $72 \mathrm{~h}$ & Total \\
\hline \multirow{3}{*}{ H. suaveolens } & $4,0 \mathrm{a} *$ & $0,5 \mathrm{a}$ & $1,75 \mathrm{a}$ & $6,25 \mathrm{a}$ \\
& {$[40]$} & {$[5,0]$} & {$[17,5]$} & {$[62,5]$} \\
M. urundeuva & $2,25 \mathrm{ab}$ & $1,5 \mathrm{a}$ & $0,0 \mathrm{a}$ & $3,75 \mathrm{ab}$ \\
& {$[22,5]$} & {$[15]$} & {$[-]$} & {$[37,5]$} \\
C. sonderianus & $1 \mathrm{bc}$ & $0,25 \mathrm{a}$ & $0,25 \mathrm{a}$ & $1,5 \mathrm{bc}$ \\
& {$[10]$} & {$[2,5]$} & {$[2,5]$} & {$[15,0]$} \\
A. fraxinifolium & $0,5 \mathrm{bc}$ & $0,75 \mathrm{a}$ & $0,0 \mathrm{a}$ & $1,25 \mathrm{bc}$ \\
& {$[5,0]$} & {$[7,5]$} & {$[-]$} & {$[12,5]$} \\
C. heliotropiifolius & $0,5 \mathrm{bc}$ & $0,25 \mathrm{a}$ & $0,25 \mathrm{a}$ & $1,0 \mathrm{bc}$ \\
& {$[5,0]$} & {$[2,5]$} & {$[2,5]$} & {$[10,0]$} \\
C. spinosa & $0,5 \mathrm{bc}$ & $0,0 \mathrm{a}$ & $0,0 \mathrm{a}$ & $0,5 \mathrm{bc}$ \\
& $0,25 \mathrm{c}$ & $0,25 \mathrm{a}$ & $1,5 \mathrm{a}$ & $2,0 \mathrm{bc}$ \\
L. microphylla & {$[2,5]$} & {$[2,5]$} & {$[15,0]$} & {$[20,0]$} \\
& $0,0 \mathrm{c}$ & $0,0 \mathrm{a}$ & $0,5 \mathrm{a}$ & $0,5 \mathrm{bc}$ \\
Controle & {$[-]$} & {$[-]$} & {$[5,0]$} & {$[5,0]$} \\
& $0,0 \mathrm{c}$ & $0,0 \mathrm{a}$ & $0,0 \mathrm{a}$ & $0,0 \mathrm{c}$ \\
& {$[-]$} & {$[-]$} & {$[-]$} & {$[-]$} \\
\hline
\end{tabular}

* Médias seguidas de mesma letra nas colunas não diferem estatisticamente entre si pelo teste de Tukey a 5\% de probabilidade. [] Eficiência.

Silva et al. (2008), ao avaliar os constituintes voláteis extraídos de folhas de plantas medicinais, verificaram que os mesmos favoreceram $100 \%$ de mortalidade de larvas de $A$. aegypti depois de 24 horas a 2.000 ppm (Hyptis fruticosa Salmz. ex Benth), 1.000 ppm (Hyptis pectinata Poit) e 300 ppm (Lippia gracilis Schauer).

Comparando-se os dados desses autores com os obtidos na presente pesquisa percebe-se que para atingir a eficiência de $100 \%$ de mortalidade com 24 horas de exposição é necessário que as larvas sejam submetidas a uma concentração maior de óleo essencial, no caso para as plantas avaliadas.

Nesta pesquisa é possível obervar que a eficiência calculada foi decrescente quando comparou-se o tratamento com óleo de $V$. arborea e os demais. Observa-se que as eficiências de $H$. suaveolens e de $M$. urundeuva foram $56,25 \%$ e $25 \%$ inferior a de $V$. arborea, respectivamente, enquanto $C$. sonderianus, $A$. fraxinifolium e $C$. heliotropiifolius foi de $12,5 \%$ e C. spinosa $6,25 \%$.

No período de 48 horas após a exposição, verificou-se que não houve diferenças significativas entre os tratamentos com óleo essencial e o tratamento controle (Tabela 1). Mesmo não diferindo estatisticamente, percebe-se que o óleo de $H$. suaveolens destaca-se dentre os demais, promovendo maior mortalidade para as larvas. Em seguida, os óleos que apresentaram melhor desempenho foram os de $C$. sonderianus e $V$. arborea. Para esse período de exposição, os óleos de $C$. 
heliotropiifolius e de L. microphylla não causaram mortalidade às larvas.

Nesse período a eficiência baseou-se na espécie $H$. suaveolens que apresentou uma porcentagem de $15 \%$ de mortalidade. A espécie $C$. sonderianus apresentou eficiência $50 \%$ menor, enquanto $V$. arborea apresentou 33,33\% e $M$. urundeuva, A. fraxinifolium e C. spinosa foi de 16,66\%.

Em estudos realizados por Kumar et al. (2011), esses autores destacaram que o óleo essencial de Menta piperita $\mathrm{L}$. possui uma excelente ação larvicida contra $A$. aegypti, tendo sua $\mathrm{CL}_{50}$ de 111,9 ppm e $\mathrm{CL}_{90}$ de 295,18 ppm após 24 horas de exposição. Quando se avaliou o período de 48 horas, observou-se que houve um aumento de $11,8 \%$ desse potencial, em comparação com o horário de 24 horas. Na presente pesquisa, para as espécies $H$. suaveolens e $C$. sonderianus (as mais representativas nesse horário) o aumento do potencial foi de 6,6 e $4 \%$, respectivamente.

Para o período de exposição de 72 horas das larvas aos óleos essenciais (Tabela 1), percebe-se que, também, nesse período como no de 48 horas não houve diferenças estatíticas. No entanto, vale ressaltar que o óleo de $V$. arborea, $C$. spinosa e A. fraxinifolium destacaram-se dos demais. Percebe-se, também, que nesse período o óleo de $L$. microphylla apresentou pela primeira vez número de larvas mortas, mesmo não diferindo estatisticamente dos demais. Com isso, verifica-se que para esse último óleo citado, nas condições avaliadas é necessário um período maior de exposição das larvas para que haja efeito cumulativo sobre as mesmas.

A eficiência desse período de exposição das larvas foi baseada na espécie $V$. arborea que apresentou $17,5 \%$ de mortalidade. A espécie C. spinosa apresentou valor porcentual inferior àquela de $85,71 \%$, enquanto $L$. microphylla foi de $28,57 \%$. M. urundeuva e A. fraxinifolium foi de $14,28 \%$.

O total de larvas mortas foi aferido no final do experimento (Tabela 1), mostrando a ação resultante dos óleos essenciais analisados. O óleo de $V$. arborea difere estatisticamente dos demais avaliados, exceto para o de $H$. suaveolens. Os tratamentos com $M$. urundeuva, $C$. sonderianus, A. fraxinifolium, C. heliotropiifolius, C. spinosa e L. microphylla não apresentaram diferenças estatíticas entre si. Todos os tratamentos, exceto V. arborea e H. suaveolens, não diferem estatisicamente da testemunha, porém, cabe ressaltar que os mesmos possuem efeitos larvicidas.

A espécie $V$. arborea apresentou a maior eficiência total com o valor de $62,5 \%$. H. suaveolens apresentou valor de eficiência de $60 \%$ menor que $V$. arborea. Enquanto $C$. spinosa, M. urundeuva, C. sonderianus, A. fraxinifolium, $C$. heliotropiifolius, L. microphylla, apresentaram valores de $32,0 \%, 24,0 \%, 20,0 \%, 16,0 \%, 8,0 \%$ e $8,0 \%$, respectivamente.

Chung et al. (2009), quando avaliaram a atividade larvicida de Dendropanax morbifera Leveille sobre A. aegypti concluíram que o óleo essencial da planta causou efeitos significativos, apresentando valores de $\mathrm{CL}_{50}$ de $62,32 \mathrm{ppm} \mathrm{e}$ $\mathrm{CL}_{90}$ de 131,21 ppm.

Estudos químicos de algumas espécies do gênero Croton mostraram que os compostos isolados exibiram elevada atividade larvicida sobre diferentes espécies de mosquitos, incluindo o $A$. aegypti. Dentre as diferentes espécies estudadas, com atividade comprovada, destacam os extratos do caule e da folha do Croton argyrophylloides, $C$. nepetaefolius, C. sonderianus e C. zehntneri (PORTO et al., 2008).

Dória et al. (2010), analisando o efeito larvicida de duas espécies de Croton do Nordeste do Brasil, concluíram que o óleo essencial de $C$. pulegiodorus e $C$. heliotropiifolius sobre larvas de A. aegypti demostraram $\mathrm{CL}_{50}$ de 159 e 544 ppm, respectivamente. Em estudos sobre a atividade larvicida de diferentes espécies de Croton sobre A. aegypti, Morais et al. (2006), destacam que as espécies $C$. zehntneri, $C$. nepetaefolius, $C$. argyrophyloides e $C$. sonderianus apresentaram valores de $\mathrm{CL}_{50}$ de 28, 84, 102 e 104 ppm, respectivamente, sobre larvas do terceiro instar.

Aproximadamente $27 \%$ das plantas estudadas por sua atividade larvicida sobre $A$. aegypti são coletadas no Brasil, das quais 77\% são coletadas no Nordeste do Brasil (SILVA et al., 2008). Isso vem destacar a grande importância da biodiversidade dessa região para os estudos com base em plantas e/ou seus derivados.

Os óleos essenciais são misturas complexas de compostos orgânicos naturais que são predominantemente compostos de terpenos (hidrocarbonetos) tais como mirceno, pineno, terpineno, limoneno, cimeno, $\alpha$ - e $\beta$ - felandreno etc.; e terpenóides (hidrocarbonetos) contendo oxigênio, tais como álcoois monoterpênicos acíclicos (geraniol, linalool), álcoois monocíclicos (metil, 4-carvomenthone, terpineol, carveol, borneol), aldeídos alifáticos (citral, citronelal, perilaldeído), fenóis aromáticos (carvacrol, timol, safrol, eugenol), álcool bicíclico (verbenol), cetonas monocíclicas (mentona, pulegona, carvona), cetonas monoterpênicas bicíclicas (tujona, verbenona, fenchona), ácidos (ácido citronélico, ácido cinâmico) e ésteres (acetato de linalilo). Alguns óleos essenciais também podem conter óxidos (1,8- cineol), constituintes que contém enxofre, antranilato de metila, cumarinas, etc. zingibereno, curcumeno, farnesol, sesquifelandreno, termerona, nerolidol, etc. (KOUL et al., 2008).

O potencial tóxico de óleos essenciais e os seus compostos contra o A. aegypti pode variar significativamente de acordo com os fatores intrínsecos e extrínsecos, espécies de plantas, partes de plantas, idade de fabricação, quimiotipos e as condições geográficas (tal como temporada de ocorrência, precipitação, porcentagem de umidade, temperatura, luz solar, e altitude), em que a planta foi recolhida, a fonte de larvas, e os métodos utilizados, em geral, para induzir diferentes respostas larvais (DIAS; MORAES, 2014).

\section{CONCLUSÕES}

Os óleos essenciais de Hyptis suaveolens e Vanillosmopsis arborea apresentam eficiência no controle de larvas de Aedes aegypti tendo potencial para serem utilizados como biolarvicidas.

\section{REFERÊNCIAS}

ABBOTT, W. S. A method for computing the effectiviness of insecticides. Journal of Economic Entomology, Maryland, v.18, n.15, p.265-267, 1925 .

ALENCAR, J. W.; CRAVEIRO, A. A.; MATOS, F. J. A. Kovatsindici as a presetion routine in mass spectra searches of volaties. Journal of Natural Products, v. 47, p. 890-892, 1984. 
AUTRAN, E. S.; NEVES, I. A.; SILVA, C. S. B.; SANTOS, G. K. N.; CÂMARA, C. A. G.; NAVARRO, D. M. A. F. Chemical composition, oviposition deterrent and larvicidal activities against Aedes aegypti of essential oils from Piper marginatum Jacq. (Piperaceae). Bioresource Technology, v. 100, n. 7, p. 2284-2288, 2009.

BARRETO, C. B.; CAVASIN, G. M.; SILVA, H. H. G.; SILVA, I. G. Estudo das alterações morfo-histológicas em larvas de Aedes aegypti (Diptera, culicidae) submetidas ao extrato bruto etanólico de Sapindus saponaria Lin (Sapindaceae). Revista de Patologia Tropical, v. 35, n. 1, p. 37-57, 2006

BRAGA, I. A.; VALLE, D. Aedes aegypti: vigilância, monitoramento da resistência e alternativas de controle no Brasil. Epidemiologia e Serviço de Saúde, v. 16, n. 4, p.295302, 2007.

CHUNG, I.; SEO, S.; KANG, E.; PARK, S.; PARK, W.; MOOND, H. Chemical composition and larvicidal effects of essential oil of Dendropanax morbifera against Aedes aegypti L. Biochemical Systematics and Ecology, v. 37, n. 1, p. 470473, 2009.

CORRÊA, J. C. R.; SALGADO, H. R. N. Atividade inseticida das plantas e aplicações: revisão. Revista Brasileira de Plantas Medicinais, v. 13, n. 4, p. 500-506, 2011.

DIAS, C. N.; MORAES, D. F. C.; Essential oils and their compounds as Aedes aegypti L. (Diptera: Culicidae) larvicides: review. Parasitology Research, v. 113, n. 1, p. 565592,2014

DÓRIA, G. A. A.; SILVA, W. J.; CARVALHO, G. A.; ALVES, P. B.; CAVALCANTI, S. C. H. A study of the larvicidal activity of two Croton species from northeastern Brazil against Aedes aegypti. Pharmaceutical Biology, v. 48, n. 6, p. 615-620, 2010.

EL-AKHAL, F.; LALAMI, A. E. O.; GUEMMOUH, R. Larvicidal activity of essential oils of Citrus sinensis and Citrus aurantium (Rutaceae) cultivated in Morocco against the malaria vector Anopheles labranchiae (Diptera: Culicidae). Asian Pacific Journal of Tropical Disease, v. 5, n. 6, p. 458-462, 2015.

FERREIRA, D. F. Sisvar: a computer statistical analysis system. Ciência e Agrotecnologia, v. 35, n. 6, p. 1039-1042, 2011.

FURTADO, R. F.; LIMA, M. G. A.; ANDRADE NETO, M.; BEZERRA, J. N. S.; SILVA, M. G. V. Atividade larvicida de óleos essenciais contra Aedes aegypti L. (Diptera: Culicidae). Neotropical Entomology, v. 34, n. 5, p. 843-847, 2005.

GERIS, R.; SILVA, I. G.; SILVA, H. H. G.; BARISON, A.; RODRIGUES FILHO, E.; FERREIRA, A. G. 2008. Diterpenoids from Copaifera reticulata Ducke with larvicidal activity against Aedes aegypti (L.) (Diptera, Culicidae). Revista do Instituto de Medicina Tropical de São Paulo, v. 50, n. 1, p. 25-28, 2008.

GUIRADO, M. M.; BICUDO, H. E. M. C. Alguns aspectos do controle populacional e da resistência a inseticidas em Aedes aegypti (Diptera, Culicidae). Bepa, v. 6, n. 64, p. 5-14, 2009.
KOUL, O.; WALIA, S.; DHALIWAL, G. S. Essential Oils as Green Pesticides: Potential and Constraints. Biopesticides International, v. 4, n. 1, p. 63-84, 2008.

KUMAR, S.; WAHAB, N.; WARIKOO, R. Bioefficacy of Mentha piperita essential oil against dengue fever mosquito Aedes aegypti L. Asian Pacific Journal of Tropical Biomedicine, v. 1, n. 2, p. 85-88, 2011.

LIMA, E. P.; OLIVEIRA FILHO, A. M.; LIMA, J. W. O.; RAMOS JÚNIOR, A. N.; CAVALCANTI, L. P. G.; PONTES, R. J. S. Resistência do Aedes aegypti ao Temefós em Municípios do Estado do Ceará. Revista da Sociedade Brasileira de Medicina Tropical, v. 39, n. 3, p. 259-263, 2006.

LIMA, G. P. G.; SOUZA, T. M.; FREIRE, G. P.; FARIAS, D. F.; CUNHA, A. P.; RICARDO, N. M. P. S.; MORAIS, S. M.; CARVALHO, A. F. U. Further insecticidal activities of essential oils from Lippia sidoides and Croton species against Aedes aegypti L. Parasitology Research, v. 112, n. 5, p. $1953-$ 1958, 2013.

MORAIS, S. M.; CAVALCANTI, E. S. B.; BERTINI, L. M.; OLIVEIRA, C. L. L.; RODRIGUES, J. R. B.; CARDOSO, J. H. L. Larvicidal activity of essential oils from Brazilian Croton species against Aedes aegypti L. Journal of the American Mosquito Control Association, v. 22, n. 1, p. 161164, 2006.

OLIVEIRA, G. L.; CARDOSO, S. K.; LARA JÚNIOR, C. R.; VIEIRA, T. M.; GUIMARÃES, E. F.; FIGUEIREDO, L. S.; MARTINS, E. M.; MOREIRA, D. L.; KAPLAN, M. A. C. 2013. Chemical study and larvicidal activity against Aedes aegypti of essential oil of Piper aduncum L. (Piperaceae). Anais da Academia Brasileira de Ciências, v. 85, n. 4, p. 1227-1234, 2013.

PORTO, K. R. A.; ROEL, A. R.; SILVA, M. M.; COELHO, R. M.; SCHELEDER, E. J. D.; JELLER, A. H. Atividade larvicida do óleo de Anacardium humile Saint Hill sobre Aedes aegypti (Linnaeus, 1762) (Diptera, Culicidae). Revista da Sociedade Brasileira de Medicina Tropical, v. 41, n. 6, p. 586-589, 2008.

PROPHIRO, J. S.; ROSSI, J. C. N.; KANI, L. A.; SANTOS, T. G.; SILVA, O. S. Estudo Comparativo do Efeito Larvicida de Extratos de Frutos Verdes e Maduros de Melia azedarach L. (Sapindales: Meliaceae) em Aedes aegypti L. (Diptera: Culicidae). BioAssay, v. 3, n. 2, p. 1-5, 2008.

PROPHIRO, J. S.; SILVA, O. S.; LUNA, J. E. D.; PICCOLI, C. F.; KANIS, L. A.; SILVA, M. A. N. Aedes aegypti and Aedes albopictus (Diptera: Culicidae): coexistence and susceptibility to temephos, in municipalities with occurrence of dengue and differentiated characteristics of urbanization. Revista da Sociedade Brasileira de Medicina Tropical, v. 44, n. 3, p. 300-305, 2011.

SILVA, W. J.; DORIA, G. A. A.; MAIA, R. T.; NUNES, R. S.; CARVALHO, G. A.; BLANK, A. F.; ALVES, P. B.; MARÇAL, R. M.; CAVALCANTI, S. C. H. 2008. Effects of essential oils on Aedes aegypti larvae: Alternatives to environmentally safe insecticides. Bioresource Technology, v. 99, n. 8, p. 3251-3255, 2008.

SRITABUTRA, D.; SOONWERA, M. 2013. Repellent activity of herbal essential oils against Aedes aegypti (Linn.) and Culex quinquefasciatus (Say.). Asian Pacific Journal of Tropical Disease, v. 3, n. 4, p. 271-276, 2013. 